\title{
Export processing zones and corporate environmental performance in emerging economies: The case of the oil, gas, and chemical sectors of Trinidad and Tobago
}

\author{
Kalim U. Shah · Jorge E. Rivera
}

Published online: 26 March 2008

(C) Springer Science+Business Media, LLC. 2008

\section{Erratum to: Policy Sci}

\section{DOI 10.1007/s11077-007-9045-8}

In the process of compressing this table for publication, coefficient values in Models 1 and 2 were shifted and hence do not correspond to the ones discussed by authors in the text of the article.

Table 4 Corporate environmental performance and firm characteristics, OLS regression models

\begin{tabular}{lll}
\hline & $\begin{array}{l}\text { Model 1 (Reference group: } \\
\text { Firms outside EPZs) }\end{array}$ & $\begin{array}{l}\text { Model 2 (Reference group: } \\
\text { Firms inside private EPZs) }\end{array}$ \\
\hline Constant & $26.156^{* * *}(6.37)$ & $29.762^{* * *}(6.64)$ \\
Location inside EPZs & $3.518^{\wedge}(1.76)$ & $1.024(0.43)$ \\
Location outside EPZs & & $9.224^{* * *}(3.25)$ \\
State EPZ & & $3.212^{* *}(3.01)$ \\
Log Size & $3.938^{* * *}(3.64)$ & $0.314(0.15)$ \\
Industry sector & $0.069(0.03)$ & $3.763^{\wedge}(1.66)$ \\
Association membership & $5.210^{*}(2.25)$ & $0.092^{* *}(2.67)$ \\
Foreign market dependence & $0.129^{* * *}(3.79)$ & $0.082^{*}(2.19)$ \\
Foreign joint venture & $0.067(1.74)$ &
\end{tabular}

The online version of the original article can be found under doi:10.1007/s11077-007-9045-8.

K. U. Shah

Department of Environmental Science and Policy, George Mason University, David King Hall, Fairfax, VA 22030, USA

e-mail: kshah2@gmu.edu

J. E. Rivera (两)

Department of Strategic Management and Public Policy, The George Washington University,

Funger Hall 615, 2201 G Street, NW Washington, DC 20052, USA

e-mail: jrivera@gwu.edu 
Table 4 continued

Model 1 (Reference group:

Firms outside EPZs)

\begin{tabular}{lll}
\hline State joint venture & $0.001(0.03)$ & $-0.002(-0.04)$ \\
Urban Community & $2.023(0.95)$ & $1.510(0.74)$ \\
$N$ & 131 & 131 \\
$F$-value & $9.380^{* * *}$ & $10.170^{* * * *}$ \\
$R^{2}$ & 0.381 & 0.431 \\
Adj.- $R^{2}$ & 0.340 & 0.388 \\
\hline
\end{tabular}

$t$-values in parentheses

*** $p<0.001$; ** $p<0.01$; * $p<0.05 ;{ }^{\wedge} p<0.1$
Model 2 (Reference group: Firms inside private EPZs)

$-0.002(-0.04)$

$1.510(0.74)$

0.43 